\title{
A clinical evaluation of random dot stereoacuity cards in infants
}

\begin{abstract}
Purpose To clinically evaluate the random dot stereoacuity cards developed by Birch and Salomao on a population of infants within the first 12 months of life and to generate baseline normative data.

Methods Random dot stereoacuity cards were constructed using vectographic vertical bar stimuli with cross disparities ranging from 45 to 1735 seconds of arc (1.65 to $3.2 \log$ seconds of arc). Wearing polarising goggles, infants were assessed using a two-alternative forced choice preferential looking staircase procedure. Success rate and stereoacuity values for performance as a function of age were evaluated.
\end{abstract}

Results One hundred and seventy-seven tests were completed on 41 infants with ages ranging from 5 to 56 weeks. The random dot stereoacuity procedure was successful in $\mathbf{1 2 8}$ (72.3\%) infants, success being dependent upon age. At 0-8 weeks of age, none of the infants produced a fixation preferance towards even the coarsest stereogram. The success rate increased to $50 \%$ at $9-16$ weeks, $97 \%$ at $17-24$ weeks, $93 \%$ at $25-36$ weeks, dropping to $69 \%$ at 37-56 weeks due to increasing intolerance to the goggles. Stereopsis was not demonstrable in infants under 8 weeks of age. By 9-16 weeks the mean stereoacuity level was $2.91 \mathrm{log}$ seconds of arc, further increasing to 2.53 at 17-24 weeks, reducing slightly to 2.65 at $25-36$ weeks. At 37-56 weeks the mean stereoacuity value was recorded as $2.53 \mathrm{log}$ seconds of arc. Conclusion Our results indicate that the infant random dot stereoacuity cards provide a simple, quick and portable test for the clinical assessment of stereopsis in infants aged between 17 and 36 weeks, and should prove to be a useful clinical tool to monitor infants at risk of binocular vision disorders.

Key words Infants, Random dot, Stereoacuity

The study of visual development in the preverbal child has been revolutionised in the last two decades by the clinical development of visual evoked potentials and behavioural techniques such as preferential looking. ${ }^{1,2}$
An improved understanding of early visual development has led to earlier intervention in paediatric eye disorders such as infantile esotropia and congenital cataract. ${ }^{3,4}$ Despite this, stereopsis in these disorders is mostly suboptimal by visual maturity. Measurement of stereoacuity in infancy may enable optimisation of treatment regimes. A similar principle has been demonstrated in the modulation of occlusion after the measurement of PL acuity in unilateral congenital cataract. ${ }^{5}$ Traditionally the measurement of stereopsis and stereoacuity in infants requires time-consuming and specialised laboratory techniques unavailable to the majority of paediatric ophthalmic clinics. Random dot stereograms were first popularised by Julesz ${ }^{6}$ and have been widely used to investigate the development of stereopsis in infants. Steroegrams with vertical line bar stimuli were used by Simons ${ }^{7}$ and Birch et al. ${ }^{8}$ to simplify the presentation of stereogram disparities. The large pattern elements of these stereograms matched infant acuity limits, while the option of presenting very small binocular disparities was retained.

Atkinson and Braddick, ${ }^{9}$ using a preferential looking technique allied with random dot stereograms generated on an oscilloscope, were able to show evidence of stereopsis in infants as young as 8 weeks.

A combination of forced choice preferential looking and visual evoked potential protocols have been used to delineate the abrupt onset of stereopsis at approximately 12 weeks of age, and its subsequent rapid development to adult levels. ${ }^{8,10}$ However most studies on the development of stereopsis have been confined to the research laboratory, where sophisticated equipment is available and the testing environment is well controlled. Birch and Salomao ${ }^{11}$ recently published laboratory results using relatively simple and portable random dot stereoacuity cards designed for infants up to the age of 24 months.

The purpose of this study was to evaluate these cards when used in NHS 'well baby' clinics or home environments and to compare the results with those obtained by Birch and Salomao. ${ }^{11}$

\author{
S.L. Calloway \\ I.C. Lloyd \\ D.B Henson \\ Manchester Royal Eye \\ Hospital \\ Manchester, UK
}

Mrs Sarah L. Calloway Orthoptic Department Royal Berkshire Hospital London Road, Reading Berkshire RG1 5AN, UK

Tel: +44 (0)1189877683

Fax: $+44(0) 1189877685$

Presented in part at the annual meeting of the Association for Research in Vision and Ophthalmology (ARVO), Fort Lauderdale, FL, USA, 10-15 May 1998

Received: 6 December 2000 Accepted in revised form: 23 April 2001 


\section{Materials and methods}

\section{Subjects}

Forty-one infants were recruited through a consecutive referral protocol with the assistance of General Practitioners and associated Health Visitors in the Cental and South Manchester Community NHS Trusts.

Referrals were taken from a wide socioeconomic background. The study was approved by Central Manchester ethics committee.

Informed consent was provided by a parent or designated guardian at the first visit. The gestation period of recruited infants ranged from 37 to 42 weeks. Infants born before 36 weeks of gestation were excluded from the study at the initial assessment. The gestational age of infants was used in the study to compensate for early developmental differences occurring in those born as early as 37 weeks, and those as late as 42 weeks gestation.

Where possible infants were seen at the following ages: 8 weeks, 12 weeks, 16 weeks, 24 weeks, 36 weeks and 52 weeks. In practice, this was not always possible due to late referral to the study (after 8 weeks), infant sleeping/waking patterns, parent work patterns and childcare commitments.

A full orthoptic assessment, non-cycloplegic refraction and fundus check were performed at the first visit to exclude infants with any ophthalmological and orthoptic abnormalities. Non-cycloplegic refraction was used to make the testing protocol as minimally invasive as possible and thus ensure adequate sample size and good compliance.

\section{Random dot stereoacuity cards}

The stereoacuity cards developed by Birch and Salomao ${ }^{11}$ consist of a set of 12 vectographic vertical bar stereograms of cross disparities. ${ }^{7}$ The dimensions of the cards, vectograph and their pattern elements are given in Fig. 1. Each set contained two stereograms with the following disparities: 1735, 865, 435, 175, 84 and 45 seconds of arc (3.2 to $1.65 \log$ seconds of arc) when used at a distance of $70 \mathrm{~cm}$.

Like Teller acuity cards, each stereogram is mounted on the left- or the right-hand side of a central grey card $(52 \mathrm{~cm}$ by $26 \mathrm{~cm})$ with a matched stereogram of zero disparity on the opposite side. A peephole in the centre of the card was added to facilitate the examiner's view of infant fixation preference. The test infant views each card while wearing polarising lenses mounted in soft frames (Brulimar Optical COMOBABY) held in place with ribbon. Testing began at card 5 (435 seconds of arc) and a two-alternative forced choice (2AFC), 2 down-1 up staircase procedure ${ }^{12}$ was performed, increasing or decreasing the presented disparities depending on the infant's responses. The session concluded when eight staircase reversals were obtained.

In order to directly compare our results to those of Birch and Salomao, ${ }^{11}$ we used the same methodology to calculate age group mean values of stereoacuity, using $\log$ seconds of arc. This involved assigning a default value of $3.5 \log$ seconds of arc ( 1 octave higher than the largest disparity of $3.2 \mathrm{log}$ seconds of arc) to infants who failed to demonstrate any stereopsis. Success rates and mean stereoacuity values were evaluated as a function of age.

\section{Results}

\section{Subjects}

The testing environment depended on the preferance of the infant's parent/guardian and was either in the NHS clinic or in the child's home. A total of 177 tests were perfomed on 41 infants (155 in the home and 22 in a clinical environment). The decision to offer parents the option of home examination was made to ensure an adequate sample size and minimise drop-out rate. The number of cases examined within a clinical setting was insufficient to draw any meaningful comparisons

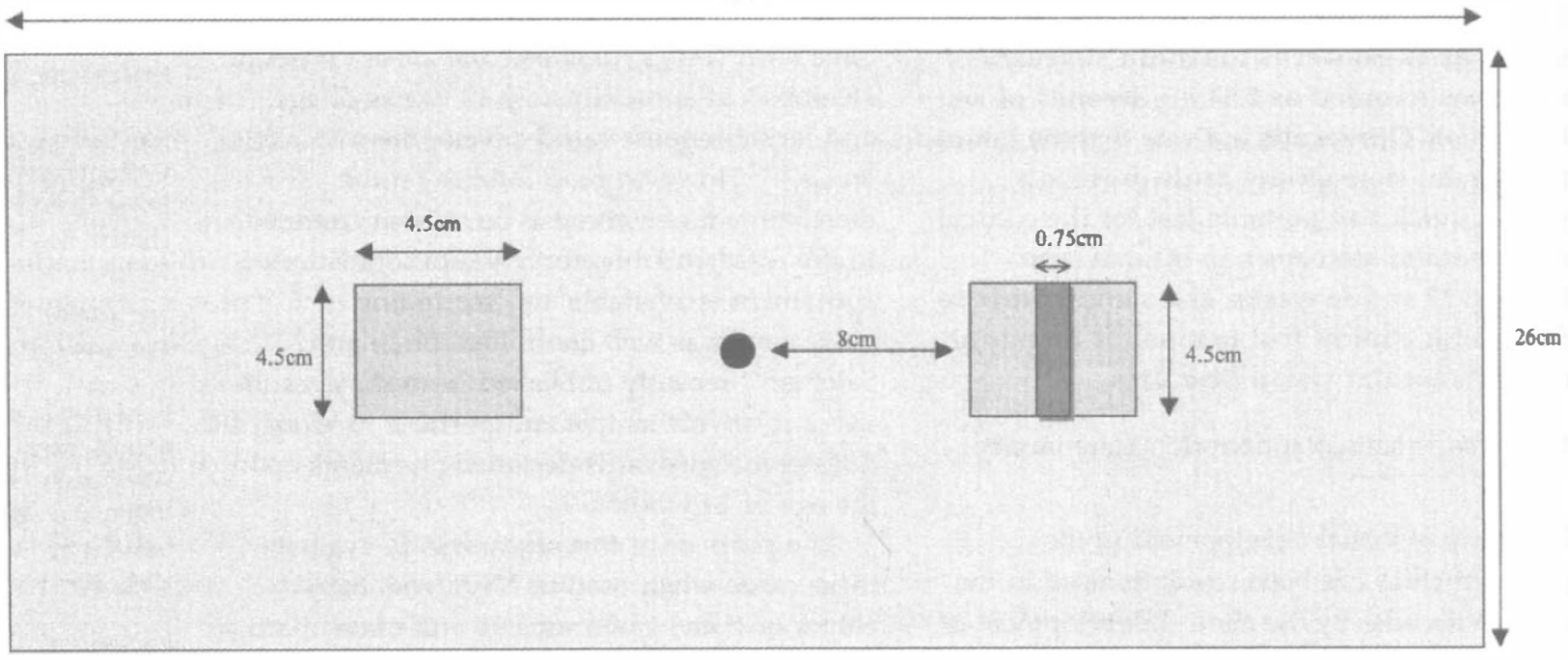

Fig. 1. Dimensions of the infant random dot stereoacuity cards and vectographs. 


\begin{tabular}{|c|c|c|c|c|c|}
\hline \multirow[b]{2}{*}{$\begin{array}{l}\text { Infant age } \\
\text { (weeks) }\end{array}$} & \multirow[b]{2}{*}{$\begin{array}{l}\text { No. of infants } \\
\text { tested }\end{array}$} & \multicolumn{2}{|c|}{ Infants who tolerated goggles (\%) } & \multirow{2}{*}{$\begin{array}{c}\text { Infants refusing to } \\
\text { wear goggles (\%) } \\
\text { Category } 3\end{array}$} & \multirow{2}{*}{$\begin{array}{c}\text { Unable to assess (\%) } \\
\text { Category } 4\end{array}$} \\
\hline & & $\begin{array}{c}\text { Positive measure of } \\
\text { stereoacuity } \\
\text { Category } 1\end{array}$ & $\begin{array}{l}\text { Negative measure of } \\
\text { stereoacuity } \\
\text { Category } 2\end{array}$ & & \\
\hline $0-8$ & 16 & 0 & 6.5 & 6.5 & 87 \\
\hline $9-16$ & 56 & 50 & 16 & 17 & 17 \\
\hline $17-24$ & 38 & 97 & 0 & 3 & 0 \\
\hline $25-36$ & 28 & 93 & 3.5 & 3.5 & 0 \\
\hline $37-56$ & 39 & 69 & 0 & 31 & 0 \\
\hline
\end{tabular}

between settings. However, it is felt that, with children of this age group, provided the clinical setting maintains a relatively distraction-free environment, very little difference will be found between results obtained in the clinical and home environments. For analysis, data were grouped into the following five age ranges: 0-8 weeks, 9-16 weeks, 17-24 weeks, 25-36 weeks and 37-56 weeks.

Each infant was assessed between 1 and 7 times. The number of assessments each child underwent was affected by the family moving out of the area, difficulties with childcare arrangements and late referral. The mean number of assessments was 4.3. The recruitment protocol led to an unequal sex distribution, with 27 males and 14 females being tested, a ratio of 2:1.

\section{Infant stereoacuity testability}

Due to the need to perform a 'screening' ophthalmological and orthoptic assessment at the first visit, the random dot stereoacuity card procedure was usually the final test performed. At subsequent visits, stereoacuity was assessed at a time when cooperation was at its maximum. Testing time was approximately 5 min, with time allowed for pacifying, if necessary.

\section{Classification of testing responses}

Category 1: Good toleration of the goggles, with a positive measurement of the infant's stereoacuity. Category 2: Good toleration of the goggles, without a positive measurement of the infant's stereoacuity. Category 3: Refusal to wear goggles, preventing successful testing.

Category 4: Unable to assess the infant due to sleepy or fractious behaviour.

Only infants who produced responses in categories 1 and 2 were included in the stereoacuity results, category 2 being allocated a score of $3.5 \log$ seconds of arc. ${ }^{11}$ Overall, $72.3 \%(128 / 177)$ of the stereoacuity test procedures (categories 1 or 2) were successful. The test results, subdivided into different age groups, are tabulated in Table 1.

\section{Infant stereoacuity values}

Mean stereoacuity values have been calculated for infants in each age group. Fig. 2 provides a graphical representation of improving stereoacuity scores in relation to infant age, with a default value of $3.5 \mathrm{log}$ seconds of arc assigned to a negative fixation response in the presence of good cooperation. ${ }^{11}$

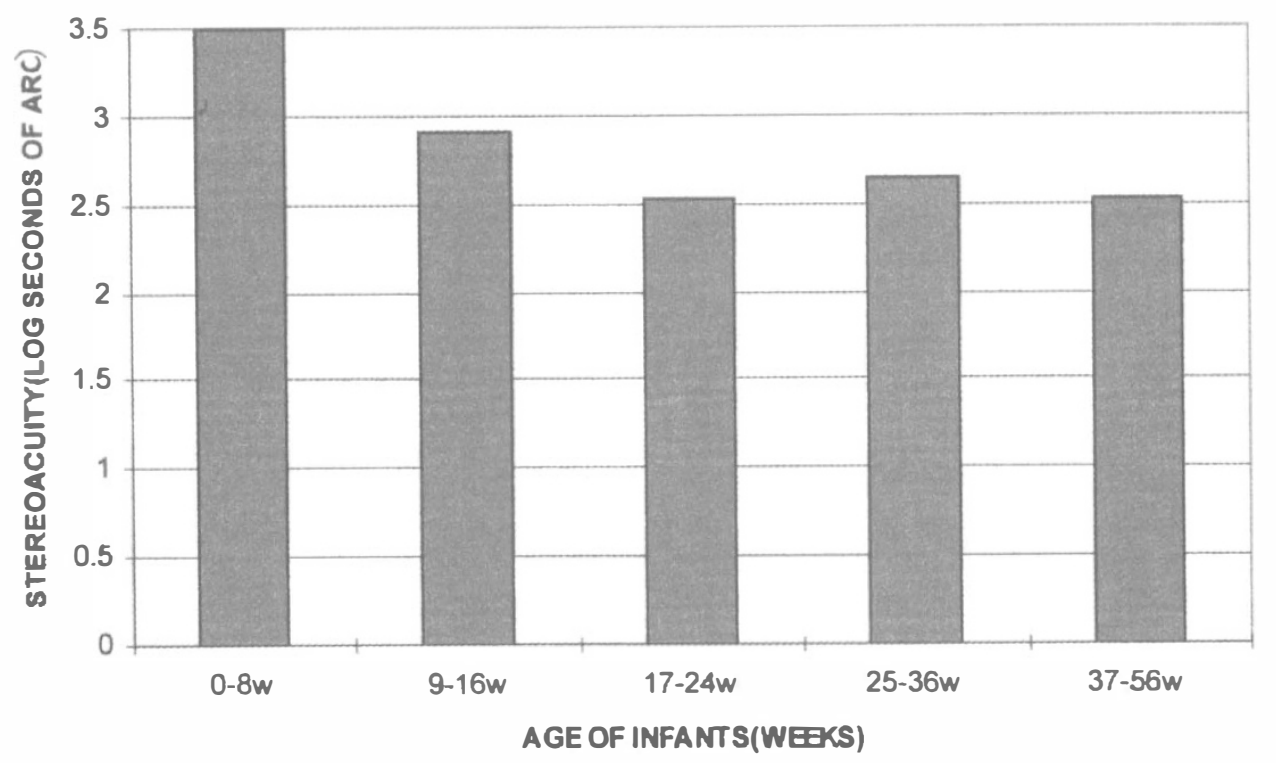

Fig. 2. Mean stereoacuity value as a function of infant age. The negative fixation response corresponds to a score of 3.5 log seconds of arc. 
The onset of measurable stereopsis occurred between 9 and 16 weeks of age in $50 \%$ of infants as a mean value of $2.91 \mathrm{log}$ seconds of arc ( 813 seconds of arc). The mean stereoacuity score increased with age to $2.53 \mathrm{log}$ seconds of arc (338 seconds of arc) at 17-24 weeks, reducing slightly to $2.65 \log$ seconds of arc (447 seconds of arc) at 25-36 weeks but subsequently returning to 2.53 (338 seconds of arc) seconds of arc between the ages of 37 and 56 weeks at a time when other studies document continued improvement. ${ }^{8,13}$ In the oldest age group, category 1 and 2 infants fell to $69 \%$ due to increased intolerance of the polarising goggles. This also explains the slight increase in mean stereoacuity in this age group when a larger improvement would be expected.

\section{Longitudinal stereoacuity data}

Longitudinal stereoacuity data of 8 infants who gave category 1 and 2 responses on five or more occasions are presented in Fig. 3. No infant showed stereopsis before the age of 8 weeks. Infants 2, 4, 6 and 7 showed gradual improvement in their stereoacuity scores throughout
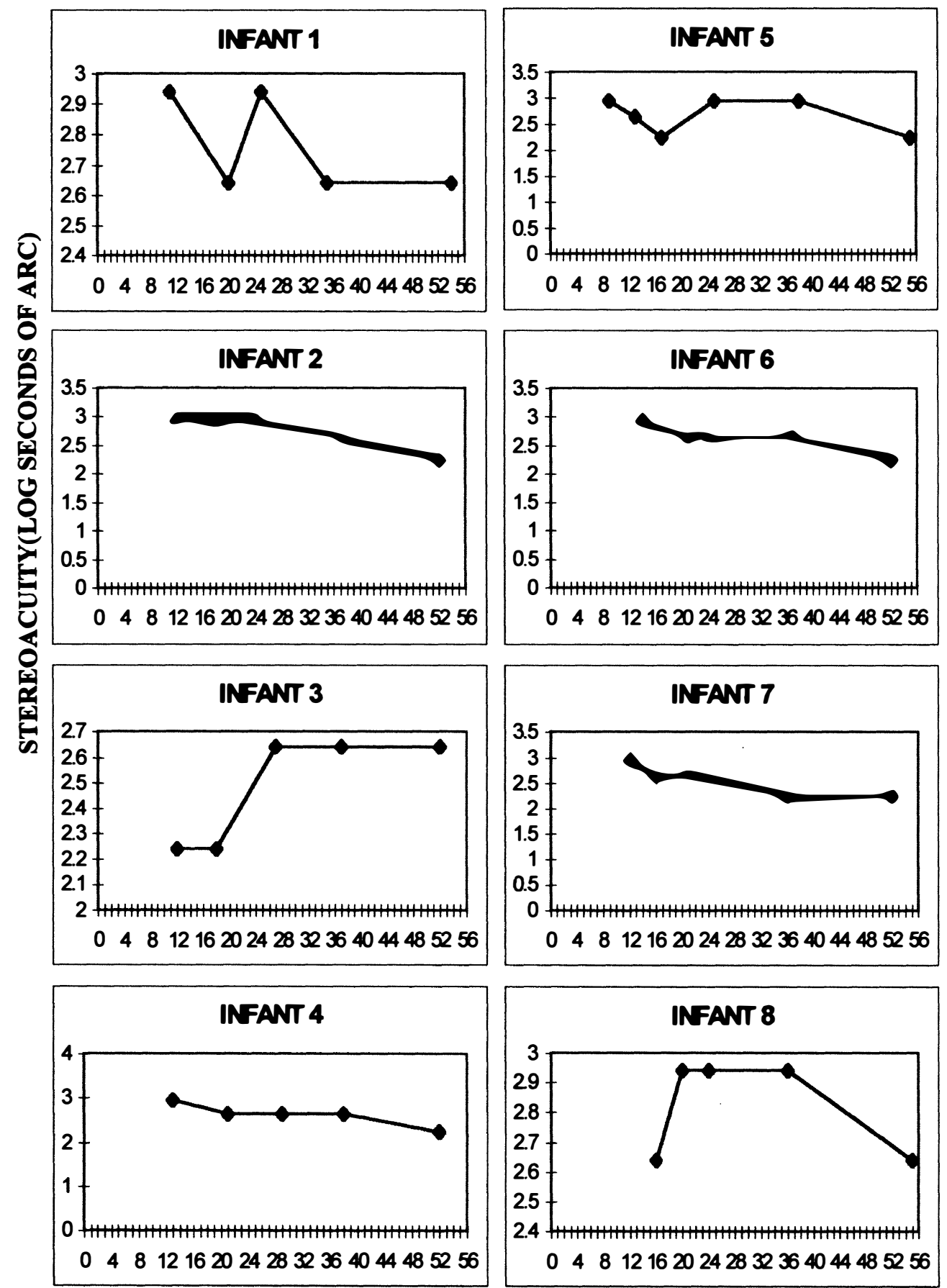

\section{AGE OF INFANTS (WETSS)}

Fig. 3. Longitudinal stereoacuity data of 8 of the study infants seen on five or more occasions. The stereoacuity values are plotted at exact infant age. 
their assessment period, whereas infants 1 and 5 produced both improvement and deterioration in their scores reflecting changes in cooperation and attention. Infants 3 and 8 produced good stereoacuity values in the first few months, but subsequently scores fell. These two infants tolerated the goggles well initially but learnt to grab the goggles and remove them. Infant 8 improved again by the end of the testing period.

\section{Discussion}

Our aim was to clinically evaluate the infant random dot stereoacuity cards in a group of normal infants within their first year.

\section{Infant stereoacuity testability}

In the study by Birch and Salomao ${ }^{11} 95$ infants (between 1.5 and 24 months of age) took part in 173 infant random dot stereotest procedures, achieving an overall success rate of $91.3 \%$. Of these 173 procedures, 15 assessments were abandoned. Seven of the 15 abandoned were due to sleepy or fussy behaviour and 1 from poor fitting goggles in the 1.5-4 month age group; the remaining 6 abandoned assessments were in the older age groups, again due to refusal to wear the goggles or fussy behaviour. In our study the success rate from testing in a clinical or home environment was $72.3 \%$ from 177 testing procedures.

Our data, from a familiar but not always distractionfree environment, suggest that the most successful age range for attempting the stereoacuity card procedure is between 17 and 36 weeks. This corresponds to the period when a rapid increase in stereoacuity is expected. ${ }^{13,14}$

As infant age increased, the percentage that failed to complete the test due to refusal to wear polarised goggles increased. In many cases this appeared to be due to a general dislike of objects on their face and the infant's increasing hand/arm strength and coordination. Occasionally, the testing distance of $70 \mathrm{~cm}$ was a problem in that infants were easily distracted by objects between themselves and the observer. A puppet screen, as typically used for preferential looking acuity cards may be helpful, but would reduce the portability and ease of the testing procedure. A closer testing distance (for example $35 \mathrm{~cm}$ ) may also be worth considering in these infants, with alternative log seconds of arc calculated for the change in testing distance.

The infant random dot stereoacuity card procedure was the final test attempted at each infant's initial assessment. This ranking in procedure order at the first visit may have led to a reduction in the percentage of infants tolerating the goggles. Unfortunately, it was not logistically possible to divide the first visit into two separate sessions, i.e. the first visit used for orthoptic/ ophthalmic assessment to decide on inclusion/exclusion within the study, and a second visit to subsequently assess stereoacuity. At further visits, the Infant Random Dot Stereoacuity card procedure was performed at a time when infant cooperation was good.

\section{Infant stereoacuity values}

Mean stereoacuity values in this study, in relation to infant age, do not compare favourably with previous studies by Birch et al. ${ }^{8}$ and Gwiazda ${ }^{13}$ and Held et al., ${ }^{14}$ using forced choice preferential looking techniques. These studies reported approximate values of 400 seconds of arc (2.60 log seconds of arc) at 3-4 months of age, improving to 60 seconds (1.78 log second of arc) at 6-12 months of age.

Birch and Salomao's ${ }^{11}$ original study of these cards recorded mean stereoacuity values of $2.76 \mathrm{log}$ seconds of arc (575 seconds of arc) at 3 months, improving to 2.45 (282 seconds of arc) at 6 months and 2.1 log seconds of arc (126 seconds of arc) by 12 months of age. These results are similar to our study up until the age of 12 months, where they found a continued improvement in stereoacuity, while our results plateaued.

Limited infant cooperation and concentration prevented re-testing. A test-retest protocol would be useful to assess the repeatability of the infant random dot stereotest in individual infants. Good repeatability of the test would reinforce the argument for adding additional intermediate steps to the procedure.

The infant random dot stereocards appear to be reliable for establishing the presence of stereopsis in infants. Random dot stereograms have an advantage over other stereoacuity tests of being free of monocular cues. The stereoacuity steps are, however, quite coarse. Calculated in seconds of arc, the lowest is 1735 seconds of arc, then increasing by 870 seconds of arc to the next card of 865 seconds of arc with jumps of 430, 260, 90 and 40 seconds of arc between subsequent stereocards. The last card (45 seconds of arc) represented the expected value of stereoacuity in a mature individual. An expansion of the number of cards at the lower end of the acuity scale may improve the ability to capture infants at early stages in the development of stereopsis. Additional cards within the present range may also capture a more accurate picture of the improvement in infant stereoacuity with age, but would also prolong testing time and reduce portability.

In conclusion, our results have shown that the infant random dot stereocard procedure is effective in assessing the presence of stereopsis in infants between the ages of 17 and 36 weeks. Below this age, infants are often too fussy or sleepy to perform the test satisfactorily. Older infants often became intolerant of the goggles, which led to test failure. An optional shorter testing distance may reduce problems with infant distraction. Despite these reservations, the results of this study performed in clinical and/or home settings compare favourably with those of Birch and Salomao, ${ }^{11}$ whose testing procedures were performed in a controlled laboratory environment.

The introduction of extra stereocard disparities both at the lower end and within the present scale could improve the precision of the acuity measurements in cooperative infants. The introduction of further cards would compromise portability to some extent, and 
would also increase testing time, but may provide information on the early stages of stereopsis and provide more precise measures of stereoacuity.

The infant random dot stereocard procedure can fulfil a useful role in paediatric opthalmology and orthoptic practice, in the assessment of infants at high risk of developing binocular visual defects, such as those with congenital cataracts, infantile strabismus or a strong family history of ocular abnormalities. The infant random dot stereocard test appears to be most successful in infants between the age of 17 and 36 weeks and may have a role in screening services.

We thank Eileen Birch, PhD of the Retina Foundation of the Southwest, Dallas, Texas for the kind donation of the stereograms used in this study and for her advice and encouragement, and the Orthoptic Department at Manchester Eye Hospital for their continued support.

\section{References}

1. Teller DY, McDonald MA, Preston K, Sebris SL, Dobson V. Assessment of visual acuity in infants and children: the acuity card procedure. Dev Med Child Neurol 1986;28:779-89.

2. Tyler CW, Apkarian P, Levi DM, Nakayama K. Rapid assessment of visual function: an electronic sweep technique for the pattern visual evoked potential. Invest Ophthalmol Vis Sci 1979;18:703-13.
3. Wright KW, Edelman PM, McVey JH, Terry AP, Lin M. High grade stereoacuity after early surgery for congenital esotropia. Arch Ophthalmol 1994;112:913-9.

4. Birch EE, Swanson WH, Stager DR, Woody M, Everett M. Outcome after very early treatment of dense congenital unilateral cataract. Invest Ophthalmol Vis Sci 1993;34:3687-99.

5. Lloyd IC, Dowler JG, Kriss A, Speedwell L, Thompson DA, Russell-Eggitt I, Taylor D. Modulation of amblyopia therapy following early surgery for unilateral congenital cataracts. $\mathrm{Br}$ J Ophthalmol 1995;79:802-6.

6. Julesz B. Binocular depth perception of computer generated patterns. Bell Syst Tech J 1960;39:1125.

7. Simons K. Stereoacuity norms in young children. Arch Ophthalmol 1981;99:439-45.

8. Birch EE, Gwiazda J, Held R. Stereoacuity development for crossed and uncrossed disparities in human infants. Vision Res 1982;22:507-13.

9. Atkinson J, Braddick O. Stereoscopic discrimination in infants. Perception 1976;5:29-38.

10. Archer S, Helveston E, Miller K, Ellis F. Stereopsis in normal infants and infants with congenital esotropia. Am J Ophthalmol 1986;101:591-6.

11. Birch EE, Salomao S, Random dot stereoacuity cards. J Pediatr Ophthalmol Strabismus 1998;35:86-90.

12. Birch EE, Hale LA. Operant assessment of stereoacuity. Clin Vision Sci 1989;4:295-300.

13. Gwiazda J. Binocular functions in human infants: correlation of stereoptic and fusion rivalry discrimination. J Pediatr Ophthalmol Strabismus 1989;26:128-33.

14. Held R, et al. Stereoacuity in human infants. Proc Natl Acad Sci USA 1980;77:5572. 